\title{
Análisis de crecimiento de epazote (Chenopodium ambrosioides L.) cultivado en invernadero
}

\author{
Mexican tea (Chenopodium ambrosioides L.) growth analysis under greenhouse conditions
}

\begin{abstract}
Cid Aguilar-Carpio', Sergio V. González-Maza², Porfirio Juárez-López²*, Irán Alia-Tejacal², Francisco Palemón-Alberto³, Yasmani R. Arenas-Julio ${ }^{4}$, Alberto S. Escalante-Estrada ${ }^{5}$

IDAGRO S de RL de CV, Carretera Yautepec-Tlayacapan, SN. Tlayacapan, Morelos, México.

2 Facultad de Ciencias Agropecuarias, Universidad Autónoma del Estado de Morelos, Cuernavaca, Morelos, México.

3 Facultad de Ciencias Agropecuarias y Ambientales, Universidad Autónoma de Guerrero. Periférico poniente s/n, Colonia Villa de Guadalupe, Guerrero. CP. 40020, Iguala, Guerrero.

4 Instituto de Ciencias Agropecuarias y Rurales. Universidad Autónoma del Estado de México. Toluca, Estado de México.

Programa de Botánica. Campus Montecillo, Colegio de Postgraduados. Km 36.5 Carretera México-Texcoco. Montecillo, Estado de México.
\end{abstract}

\section{ABSTRACT}

The Mexican tea (Chenopodium ambrosioides L.) is an aromatic and medicinal plant. Information on its growth dynamics and mineral nutrition for its production is insufficient, therefore, the objective of the study was to evaluate the effect of three concentrations of the Steiner nutrient solution on the growth and production of Mexican tea grown under greenhouse conditions. The study was conducted in Cuernavaca, Morelos, Mexico; with the genotype H-1000 (Hortaflor $\left.{ }^{\circledR}\right)$ grown in tezontle as substrate. Three concentrations (50, 75 and $100 \%$ ) of the Steiner nutrient solution were applied to evaluate the growth of the crop by means of destructive samplings at $39,46,53,60,67$ and 74 days after sowing; in each sampling, the following were determined: plant height, leaf area, leaf area index (LAl), weight of fresh matter, weight of dry matter, crop growth rate (CGR), absolute growth rate (AGR), and production per plant. A regression analysis was performed on the variables with the Excel ${ }^{\oplus}$ program, and for the case of production per plant, an analysis of variance and Tukey's mean test $(P \leq 0.05)$ were performed. The highest height and production in the Mexican tea crop grown in a greenhouse and with inorganic substrate was obtained with Steiner's nutrient solution at $100 \%$, which coincided with the highest CGR, AGR and LAl values, and whose equations were adjusted to a quadratic model. The results obtained suggest the use of Steiner's $100 \%$ nutrient solution to obtain the best productivity in this aromatic and medicinal plant.

Keywords: crop nutrition, dry matter, yield, medicinal plant.

\section{RESUMEN}

El epazote (Chenopodium ambrosioides L.) es una planta aromática y medicinal. La información sobre su dinámica de crecimiento y nutrición mineral para su producción es insuficiente, por lo que, el objetivo del estudio fue evaluar el efecto de tres concentraciones de la solución nutritiva Steiner sobre el crecimiento y producción de epazote cultivado en invernadero. El estudio se realizó en Cuernavaca, Morelos, México; con el genotipo $\mathrm{H}-1000$ (Hortaflor $^{\circledR}$ ) cultivado en tezontle como sustrato. Se aplicaron tres concentraciones (50, 75 y $100 \%$ ) de la solución nutritiva Steiner para evaluar

*Autor para correspondencia: Porfirio Juárez-López

Correo electrónico: porfirio.juarez@uaem.mx

Recibido: 15 de noviembre de 2020

Aceptado: 7 de marzo de 2021 el crecimiento del cultivo mediante muestreos destructivos a los $39,46,53,60,67$ y 74 días después de la siembra; en cada muestreo se determinó: altura de planta, área foliar, índice de área foliar (IAF), peso de materia fresca, peso de materia seca, tasa de crecimiento del cultivo (TCC), tasa absoluta de crecimiento (TAC) y producción por planta. A las variables se les realizó un análisis de regresión con el programa Excel ${ }^{\circledR}, y$ para el caso de producción de epazote por planta se realizó análisis de varianza y prueba de medias de Tukey $(P \leq 0.05)$. La mayor altura y producción en el cultivo de epazote cultivada en invernadero y con sustrato inorgánico se obtuvo con la solución nutritiva de Steiner a $100 \%$, lo cual coincidió con los valores más altos de la TCC, la TAC y el IAF, y cuyas ecuaciones se ajustaron a un modelo cuadrático. Con los resultados obtenidos, se sugiere emplear la solución nutritiva de Steiner a $100 \%$ para obtener la mejor productividad en esta planta aromática y medicinal.

Palabras clave: nutrición de cultivos, materia seca, rendimiento, planta medicinal.

\section{INTRODUCCIÓN}

El crecimiento es un incremento irreversible en el tamaño de las plantas el cual a menudo es acompañado por cambios en la forma, es decir, es un aumento constante en el tamaño acompañado de procesos como la morfogénesis y la diferenciación celular. El crecimiento es un proceso fisiológico complejo, que depende de la fotosíntesis, la respiración, la división celular, la elongación, la diferenciación, entre otros, y que además está influenciada por factores como temperatura, intensidad de luz, densidad de población, y disponibilidad de agua y de nutrimentos (Taiz et al., 2014).

El análisis de crecimiento usa medidas directas tales como material seca de la planta, área foliar total y tiempo; y medidas derivadas que pueden ser obtenidas a partir de las medidas directas, como la tasa de crecimiento del cultivo (TCC), que es un índice de la productividad agrícola el cual mide la ganancia en peso de un cultivo por unidad de área de suelo y por unidad de tiempo; el índice del área foliar (IAF), que representa la relación entre el área foliar y el área de suelo ocupada por el cultivo; así como la tasa absoluta 
de crecimiento (TAC) que se refiere al incremento en peso seco de la planta por unidad de tiempo (Escalante-Estrada y Kohashi-Shibata, 2015; Santos-Castellanos et al., 2010).

Por otro lado, el epazote (Chenopodium ambrosioides L.) es una planta aromática y medicinal nativa de Mesoamérica y Sudamérica que se utiliza como alimento y condimento. La palabra "epazote" proviene del Nahuatl epatl, hierba fétida y tzotl dulce, debido al olor que emana esta planta y que son originados por monoterpenoides, sesquiterpenoides y flavonoides (Blanckaert et al., 2012; Jaramillo et al., 2012). La importancia de estudiar plantas medicinales es que tienen propiedades antioxidantes que pueden favorecer la salud humana (Graciano-Cristóbal et al., 2020; Vidal-Gutiérrez et al., 2020). En México, en el año 2019 se cosecharon 166.1 ha de epazote fresco con una producción anual de 1,833.7 t y un rendimiento promedio de $11.04 \mathrm{t} \mathrm{ha}^{-1}$; los principales estados productores fueron Puebla, Tlaxcala y Estado de México (SIAP, 2020).

Existen estudios en epazote que se enfocan en la morfología, histología, actividad toxicológica y farmacológica de la planta (Blanckaert et al., 2011; Gómez-Castellanos, 2008; Nascimento et al., 2006), así como en la bioactividad del aceite esencial del epazote en hongos fitopatógenos (Jaramillo et al., 2012), sin embargo, no se encontraron reportes en la literatura acerca de estudios de análisis de crecimiento en este cultivo.

En adición a lo anterior, la mayoría de los estudios de análisis de crecimiento se han enfocado principalmente en cereales como maíz (Aguilar-Carpio et al., 2015; SánchezTorres et al., 2012) y trigo (Hernández-Córdova et al., 2015); también en hortalizas como tomate rojo (Barraza et al., 2015), uchuva (Aguilar-Carpio et al., 2018), chile jalapeño (Azofeifa y Moreira, 2004), papa (Santos-Castellanos et al., 2010), acelga, betabel, cebolla, espinaca, lechuga, pepino y zanahoria (Barrientos-Llanos et al., 2012), pero hacen falta estudios que consideren plantas aromáticas y medicinales, como es el caso del epazote y que además asocien la nutrición mineral con el análisis de crecimiento del cultivo, por lo que el objetivo del presente estudio fue evaluar el efecto de tres concentraciones de la solución nutritiva Steiner sobre el crecimiento y producción en el cultivo de epazote en condiciones de invernadero.

\section{MATERIALES Y MÉTODOS}

El estudio se realizó en Cuernavaca, Morelos, México $\left(18^{\circ} 58^{\prime} 51^{\prime \prime}\right.$ latitud norte y $99^{\circ} 13^{\prime} 55^{\prime \prime}$ longitud oeste a 1866 msnm), de abril a julio de 2018. El 28 de abril se sembraron semillas de epazote del genotipo $\mathrm{H}-1000$ de la empresa Hortaflor las cuales se colocaron cinco semillas por cada maceta. La emergencia ocurrió a los 5 días después de la siembra (dds), y a los 14 días se realizó un raleo para finalmente dejar una planta por maceta.

Los tratamientos fueron concentraciones: 50,75 y $100 \%$ de la solución nutritiva de Steiner (1984) (Tabla 1). El diseño experimental fue completamente al azar con nueve repeticiones. La unidad experimental estuvo conformada por
Tabla 1. Composición química de las tres concentraciones (tratamientos) de la solución nutritiva Steiner.

Table 1. Chemical composition of the three Steiner nutrient solution concentrations (treatments).

\begin{tabular}{lcccccc}
\hline $\begin{array}{l}\text { Concentración } \\
\text { de la } \\
\text { solución } \\
\text { nutritiva (\%) }\end{array}$ & $\mathrm{Ca}^{2+}$ & $\mathrm{K}^{+}$ & $\mathbf{M g}^{2+}$ & $\mathbf{N O}_{3}{ }^{-}$ & $\mathbf{H}_{2} \mathbf{P O}_{4}^{-}$ & $\mathbf{S O}_{4}{ }^{2-}$ \\
\cline { 2 - 7 } & \multicolumn{5}{c}{ mEq L-1 $^{-1}$} \\
\hline 50 & 4.5 & 3.50 & 2.0 & 6.0 & 0.50 & 3.50 \\
75 & 6.25 & 5.25 & 3.0 & 9.0 & 0.75 & 5.25 \\
100 & 9.00 & 7.00 & 4.0 & 12.0 & 1.00 & 7.00 \\
\hline
\end{tabular}

seis macetas de polietileno color negro de $5 \mathrm{~L}$ de capacidad, llenadas con tezontle como sustrato, con granulometría de 1 a $8 \mathrm{~mm}$.

Los fertilizantes que se usaron para preparar la solución nutritiva fueron nitrato de calcio, nitrato de potasio, sulfato de magnesio, sulfato de potasio y fosfato monopotásico. Los microelementos se aplicaron mediante mezcla comercial de quelatos (Ultrasol Micro Mix, $\mathrm{SQM}^{\circ}$ ), en dosis de $20 \mathrm{~g}$ por cada $500 \mathrm{~L}$ de solución nutritiva. Para calcular esta dosis se consideró el suministro de 3 ppm de hierro (Fe), ya que la mezcla comercial de micronutrimentos contiene las siguientes concentraciones en porcentaje: $\mathrm{Fe}$ (7.5), $\mathrm{Mn}$ (3.7), $\mathrm{B}(0.4), \mathrm{Zn}(0.6), \mathrm{Cu}(0.3)$, y Mo (0.2). El pH se ajustó entre 5.5 y 6.0 con ácido sulfúrico. La conductividad eléctrica fue de $1.0,1.5$ y $2.0 \mathrm{dS} \mathrm{m}^{-1}$, para las concentraciones 50,75 y $100 \%$, respectivamente. El riego se realizó diariamente y el aplicado varió 0.25 a $1.5 \mathrm{~L}$ por macera en función del crecimiento del cultivo.

Durante el desarrollo del estudio se registró semanalmente la temperatura media $\left({ }^{\circ} \mathrm{C}\right)$ y humedad relativa (\%) dentro del invernadero con un registrador de datos ambientales Hobo Modelo U12 (Onset').

El crecimiento del cultivo se evaluó en función de las tres concentraciones de la solución nutritiva de Steiner: 50, 75 y $100 \%$, mediante muestreos destructivos de nueve plantas por tratamiento a los $39,46,53,60,67$ y 74 días después de la siembra (dds); en cada muestreo se midió, la altura de la planta desde la base del tallo hasta el meristemo apical y el área foliar, el cual se determinó con un medidor de área foliar (LI-COR 3100 ). Con estos datos se calculó el índice de área foliar (IAF), con base en la siguiente ecuación:

$$
\overline{\mathrm{IAF}}=\frac{\left(\mathrm{AF}_{2}-\mathrm{AF}_{1}\right)}{A}
$$

Dónde: $\mathrm{AF}=$ área foliar $\left(\mathrm{cm}^{2}\right)$ y $\mathrm{A}$ representa el área ocupada por la planta (Rakesh et al., 2017).

También se evaluó el peso de materia fresca ( $\mathrm{g}$ por planta), la cual estuvo constituida por la biomasa fresca aérea y se cuantificó con una báscula digital $\left(\mathrm{OHAUS}^{\circ}\right)$, con aproximación de $0.0001 \mathrm{~g}$; asimismo, para el peso de materia seca por planta (MS, g por planta), las muestras que se usaron para peso de materia fresca se secaron a $70^{\circ} \mathrm{C}$ en una estufa de circulación de aire forzado hasta peso constante. Con 
estos datos se calcularon las tasas medias de: crecimiento del cultivo (TCC) y absoluta de crecimiento (TAC) mediante las ecuaciones:

$$
\overline{T C C}=\frac{1}{A} *\left(\frac{P S_{2}-P S_{1}}{T_{2}-T_{1}}\right)
$$

Dónde: $\mathrm{PS}_{2}$ y $\mathrm{PS}$ indican el peso de la materia seca de la planta en los tiempos $\mathrm{T}_{2} y \mathrm{~T}_{1}$, respectivamente y $A\left(227 \mathrm{~cm}^{2}\right)$ representa el área ocupada por la planta (Aguilar-Carpio et al., 2018).

$$
\overline{T A C}=\frac{P S_{2}-P S_{1}}{T_{2}-T_{1}}
$$

Dónde: $\mathrm{PS}_{2}$ y $\mathrm{PS}_{1}$ indican el peso de la materia seca de la planta en los tiempos $\mathrm{T}_{2}$ y $\mathrm{T}_{1}$, respectivamente (EscalanteEstrada y Kohashi-Shibata, 2015).

Adicionalmente, para determinar la producción de epazote en g por planta, se consideró el peso de materia fresca en el último muestreo, el cual fue a los 74 días después de la siembra (dds).

\section{Análisis estadístico}

A las variables en estudio se les realizó un análisis de regresión y se obtuvo la ecuación correspondiente, con el programa Excel ${ }^{\circ}$, Microsoft Office de Windows. Para la variable producción de epazote por planta se realizó un análisis de varianza y prueba de medias de Tukey $(P \leq 0.05)$ con el programa estadístico de SAS (Statistical Analysis System) versión 9.1 (SAS Institute, 2004).

\section{RESULTADOS Y DISCUSIÓN Temperatura y humedad relativa}

La media semanal de la temperatura y humedad relativa durante el desarrollo del cultivo que fluctuó entre 19.1 a $24.6{ }^{\circ} \mathrm{C}$ y 50.3 a $82.7 \%$, respectivamente (Figura 1). Estas condiciones ambientales son aproximadas a las de Huidobro-Medina (2013), quien al cultivar epazote en un sistema hidropónico en condiciones de invernadero, reportaron que la temperatura media osciló entre 18 y $26^{\circ} \mathrm{C}$ y la humedad relativa entre 60 y $75 \%$, en el Estado de México. Se ha re-

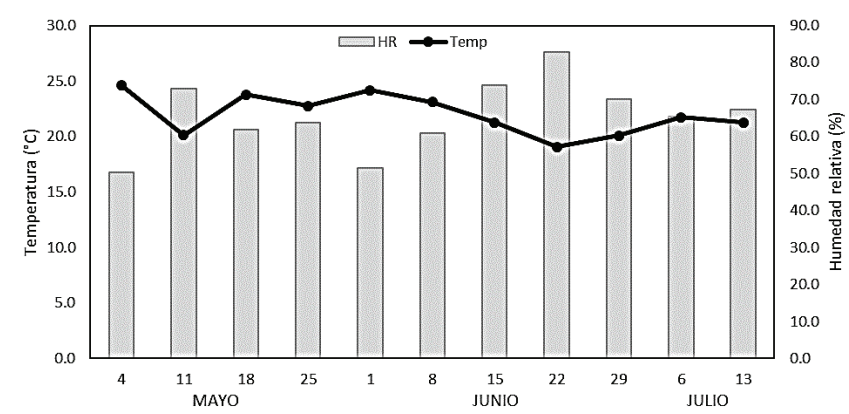

Figura 1. Promedios semanales (7 días) de temperaturas (Temp) y humedad relativa (HR) durante el desarrollo del cultivo de epazote.

Figure 1. Weekly ( 7 days) averages of temperature and relative humidity during Mexican tea crop development. portado que el epazote se adapta a distintos hábitats sobre todo a regiones templadas, semicálidas y cálidas (HuidobroMedina, 2013; Méndez-Isidro, 2014), lo que permite que las especies de este género se propague y crezca con facilidad en diversos ambientes.

\section{Altura de la planta}

A los 74 dds, la concentración de la solución nutritiva de Steiner al $100 \%$ generó la mayor altura de planta $(64.7 \mathrm{~cm})$ con un incremento por día de $3.20 \mathrm{~cm}$ en comparación con la concentración de $75 \%(59.2 \mathrm{~cm})$ y un incremento por día de $3.13 \mathrm{~cm}$ (Figura 2). Es importante señalar, que la solución nutritiva de Steiner al $100 \%$ desde los 46 dds fue superior a la concentración del $50 \%$, la cual generó una altura de 55.5 $\mathrm{cm}$ a los $74 \mathrm{dds}$. Esto nos indica que la altura de la planta está relacionada con la aportación de los nutrimentos esenciales aplicados en el sustrato, lo que posiblemente promovió un aumento en el alargamiento y división celular (Azcón-Bieto y Talón, 2008). Huidobro-Medina (2013) reportó altura promedio de $62 \mathrm{~cm}$ en plantas de epazote a los 75 dds en condiciones de invernadero. Por otro lado, Blanckaert et al. (2011) encontraron que la altura de epazote osciló de 59 a $109 \mathrm{~cm}$, en etapa vegetativa antes de la floración. En otros cultivos herbáceos, se ha reportado la misma tendencia que la observada en el presente estudio; al respecto, Cruz-Crespo et al. (2017) al evaluar concentraciones de la solución nutritiva de Steiner de 25 a $100 \%$ en cilantro a los 50 ddt, reportaron un incremento en altura de 38.9 a $56.8 \mathrm{~cm}$.

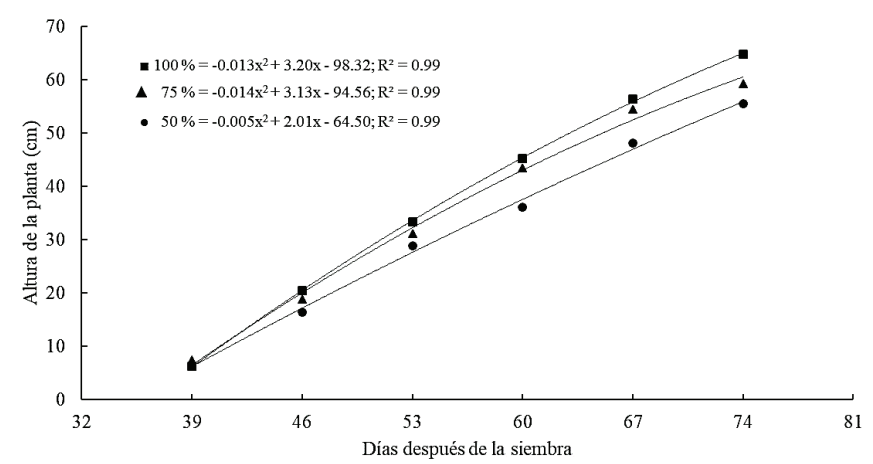

Figura 2. Efecto de la solución nutritiva Steiner en la altura de la planta de epazote.

Figure 2. Effect of Steiner nutrient solution on the Mexican tea plant height.

\section{Materia seca, tasa de crecimiento del cultivo (TCC) y tasa} absoluta de crecimiento (TAC)

En cuanto a la materia seca, la concentración al 100 $\%$ presentó el valor más alto (8.42 $\mathrm{g}$ por planta) a los $74 \mathrm{dds}$, en comparación a la solución a 75 y 50 \% (Figura 3). Durante el desarrollo del cultivo, la materia seca por planta presentó un crecimiento progresivo en función del incremento de la solución nutritiva. Las tendencias de la materia seca en las tres concentraciones se ajustaron a un modelo cuadrático. Así, la producción de materia seca por planta por día fue de $0.25 \mathrm{~g}$ para la concentración de $100 \%$, el cual superó a las concentraciones de 75 y $50 \%$ en 0.14 y 0.07 g, respectivamente. Lo anterior, indica que la generación de materia seca 


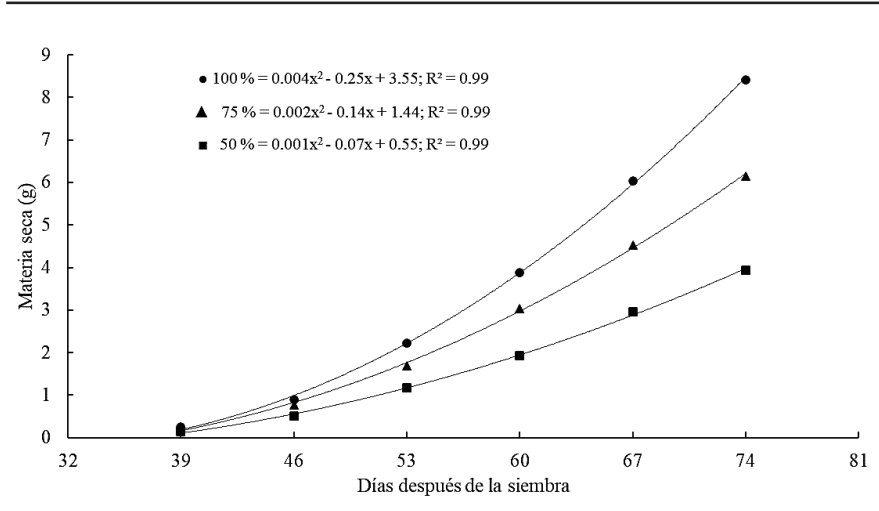

Figura 3. Producción de materia seca del cultivo de epazote en función de la concentración de la solución nutritiva Steiner.

Figure 3. Dry matter production of Mexican tea crop as a function of the Steiner nutrient solution concentration.

está directamente relacionada con la disponibilidad y aprovechamiento de los nutrimentos en el suelo o en el medio de cultivo, con lo cual, resulta una mejor actividad fotosintética (Azcón-Bieto y Talón, 2008). Al respecto, Huidobro-Medina (2013) reportaron materia seca de $9.7 \mathrm{~g}$ de epazote a los 75 dds, esos resultados son aproximados a los obtenidos con la concentración de $100 \%$ del presente estudio. Blanckaert et al. (2011) reportaron valores mayores de materia seca de epazote $(18.18 \mathrm{~g})$, esto posiblemente a las condiciones en que se desarrolló el cultivo, así como del genotipo utilizado. Los resultados obtenidos son similares a los reportados por Cruz-Crespo et al. (2017), quienes encontraron que la solución nutritiva Steiner a 100 \% de concentración generó el mayor peso seco en plantas de cilantro, durante su crecimiento.

En la tasa de crecimiento del cultivo (TCC) se observó un aumento diferencial por efecto de las tres concentraciones aplicadas (Figura 4). Al igual que las variables mencionadas previamente, estos incrementos se ajustaron a un modelo cuadrático. La concentración a 100 \% causó una mayor producción de materia seca por área sembrada por día $(5.73 \mathrm{~g}$ $\mathrm{cm}^{-2}$ día $\left.{ }^{-1}\right)$, respecto a la concentración al $75 \%\left(5.24 \mathrm{~g} \mathrm{~cm}^{-2}\right.$ día ${ }^{-1}$ y $50 \%$ (3.66 $\mathrm{g} \mathrm{cm}^{-2}$ día $\left.^{-1}\right)$, lo que puede atribuirse a una mejor eficiencia de la planta para producir materia seca con la solución nutritiva de Steiner a $100 \%$. En la concentración a $100 \%$, la TCC fue superior desde los 46 dds, respecto a la

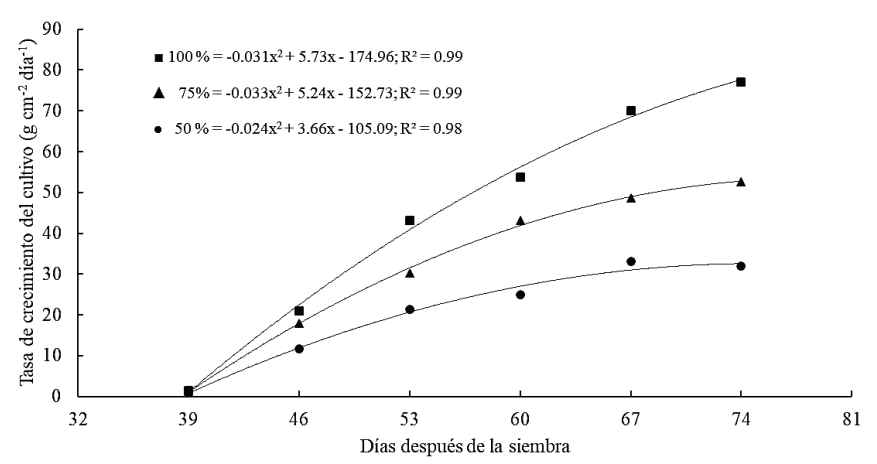

Figura 4. Dinámica de la tasa de crecimiento del cultivo de epazote en función de la concentración de la solución nutritiva Steiner.

Figure 4. Crop growth rate dynamics of Mexican tea crop as a function of the Steiner nutrient solution concentration. de 50 \%. La máxima formación de materia seca se observó a los 74 dds con $77.14 \mathrm{~g} \mathrm{~cm}^{-2}$ día $^{-1}$ con la concentración al $100 \%$. En la literatura no se encontraron reportes de tasas de crecimiento del cultivo en epazote, sin embargo, Del Pozo et al. (2001) obtuvieron mayor TCC con la aplicación nitrógeno en pasto estrella (Cynodon nlemfuensis); esta tendencia es similar en los resultados en el presente estudio, ya que al incrementar el suministro de nutrimentos se estimuló de forma positiva la velocidad de crecimiento del cultivo.

En la tasa absoluta de crecimiento se observó que la más alta acumulación de materia seca por día se presentó a los 74 dds (Figura 5), con la solución nutritiva de Steiner al $100 \%\left(0.340 \mathrm{~g} \mathrm{día}^{-1}\right)$, en comparación con la concentración al $75 \%\left(0.232 \mathrm{~g} \mathrm{día}^{-1}\right)$ y al $50 \%\left(0.140 \mathrm{~g} \mathrm{día}^{-1}\right)$. La TAC se ajustó a un modelo polinómico de segundo grado, donde con la concentración al 100 \% se incrementó el peso de la materia seca en $0.025 \mathrm{~g} \mathrm{día}^{-1}$, lo que indica una mayor acumulación de materia seca, debido a un mejor aprovechamiento de los recursos nutrimentales, y esto a su vez, se relaciona con un incremento en el dosel vegetal.

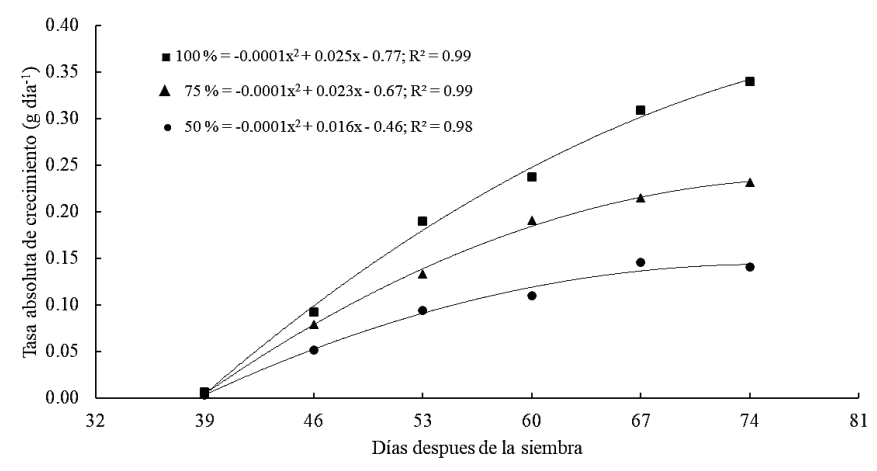

Figura 5. Dinámica de la tasa absoluta de crecimiento del cultivo de epazote en función de la concentración de la solución nutritiva Steiner.

Figure 5. Absolute growth rate dynamics of the Mexican tea crop as a function of the Steiner nutrient solution concentration.

\section{Área foliar e índice de área foliar}

El área foliar se afectó positivamente a medida que se aumentaron las concentraciones de la solución nutritiva (Figura 6). Esta respuesta en las tres soluciones se ajustó a un modelo cuadrático. Puede apreciarse que, en la concentración a $100 \%$, se produjo la mayor área foliar por día $\left(5.92 \mathrm{~cm}^{2}\right.$ día $\left.{ }^{-1}\right)$, respecto a la concentración a $75 \%\left(3.72 \mathrm{~cm}^{2}\right.$ día $\left.^{-1}\right)$ y a $50 \%\left(1.02 \mathrm{~cm}^{2}\right.$ día $\left.^{-1}\right)$. Esta respuesta está asociada al estado nutrimental de la planta, el cual promovió un aumento en el aparato fotosintético, y en consecuencia una mayor formación de hojas. Cabe señalar, que la máxima expansión foliar se pudo observar a los 74 dds con la solución nutritiva al 100 $\%\left(1,011.9 \mathrm{~cm}^{2}\right)$. Estos resultados coinciden con lo reportado por Huidobro-Medina (2013) quien al aplicar la solución nutritiva Hidro-FESI encontraron área foliar promedio de $992 \mathrm{~cm}^{2}$ en plantas de epazote cultivadas en condiciones de invernadero.

En cuanto al índice de área foliar (IAF), al aplicar la concentración a $100 \%$ se observó un IAF de 1.0 a los 74 dds, siendo el más alto en comparación a las concentraciones de 


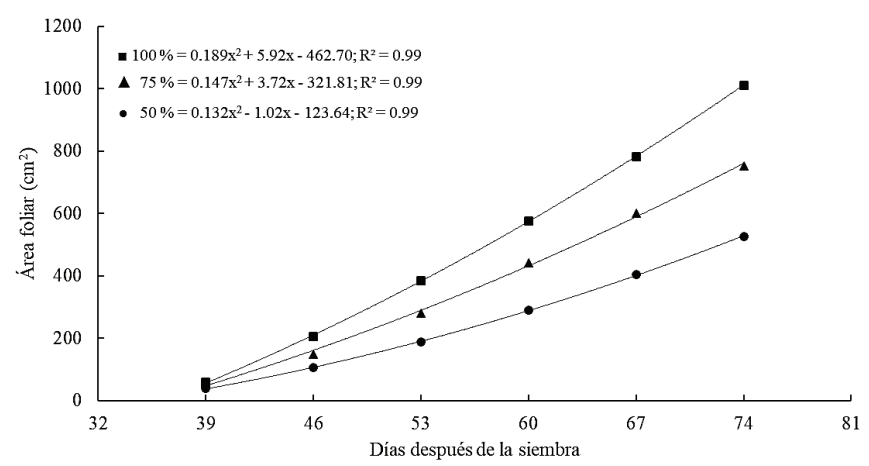

Figura 6. Efecto de la solución nutritiva Steiner en el área foliar en el cultivo de epazote.

Figure 6. Effect of Steiner nutrient solution on leaf area in the Mexican tea crop.

$75 \%$ y de $50 \%$ (Figura 7). Los menores IAF durante el ciclo de cultivo fueron obtenidos con la solución Steiner al $50 \%$, donde el IAF fue de 0.5 desde los 60 hasta los 74 dds, la concentración y periodo antes mencionado fue superado por la solución nutritiva al 100\% con un IAF (0.6) desde los 53 dds, lo que indica que la disponibilidad de los nutrimentos favoreció el incremento en la producción del área foliar por unidad de superficie de suelo en un periodo más corto. Fghire et al. (2017) en plantas de Chenopodium quinoa reportó IAF entre 1.9 a 3.13 en diferentes genotipos, estas diferencias se deben principalmente a los genotipos utilizados.

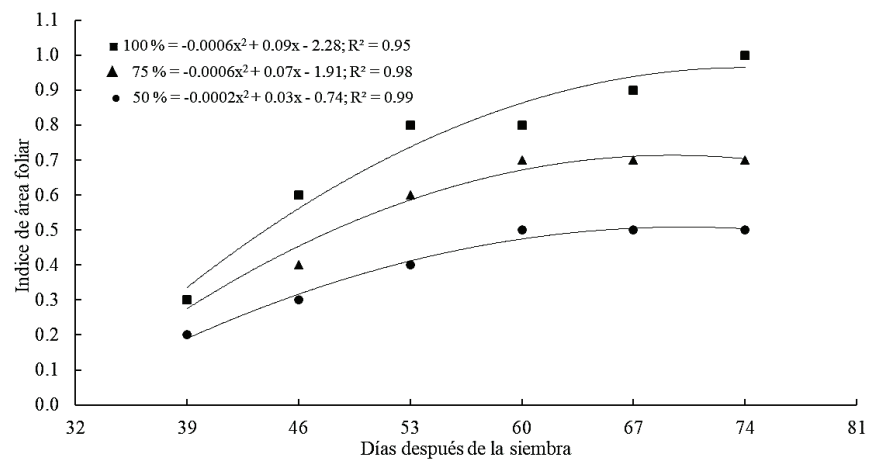

Figura 7. Índice de área foliar en el cultivo de epazote en función de la concentración de la solución nutritiva Steiner.

Figure 7. Leaf index area of the Mexican tea crop as a function of the Steiner nutrient solution concentration.

\section{Peso materia fresca}

El valor más alto de eso de materia fresca, (Figura 8), se obtuvo a los 74 dds con la concentración de $100 \%$ (71.2 g por planta), seguido de la concentración al 75 \% (54.1 g por planta) y la de $50 \%$ (34.7 g por planta). Esto indica que a medida que se incrementó la concentración de la solución nutritiva también se obtuvo una mejor producción en el peso fresco del cultivo. Dicha respuesta está determinada por un aumento en el dosel vegetal estimulado por la solución Steiner que produce un efecto en la expansión foliar. Al respecto, CruzCrespo et al. (2017) indican que a medida que se incrementa la concentración de la solución Steiner también aumenta la materia fresca en plantas de cilantro, y reportaron la mayor biomasa fresca con la concentración al $100 \%$, a los 90 dds. En

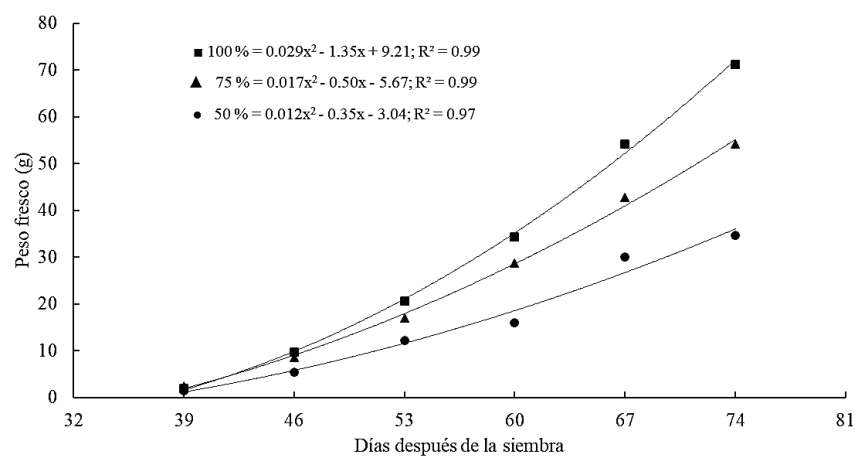

Figura 8. Peso fresco del cultivo de epazote en función de la concentración de la solución nutritiva Steiner.

Figure 8. Fresh weight of the Mexican tea crop as a function of the Steiner nutrient solution concentration.

contraste, Juárez-Rosete et al. (2019) encontraron que al aplicar el $100 \%$ de la solución Steiner, el peso fresco en orégano disminuyó en comparación al $75 \%$, lo cual fue atribuido a una excesiva nutrición que induce limitaciones de absorción nutrimental, lo que dificulta la absorción de iones por la raíz. Estos resultados confirman que las concentraciones de la solución nutritiva pueden afectar de manera diferencial el crecimiento de las plantas, en función de la especie.

En general, con las concentraciones de la solución nutritiva a $100 \%$ se obtuvieron los mejores resultados en todas las variables evaluadas, en comparación a la concentración a 75 y $50 \%$. Este comportamiento puede deberse a que la concentración de $\mathrm{N}$-nitrato corresponde a 6,9 y $12 \mathrm{mEq} \mathrm{L}^{-1}$ en las concentraciones de la solución a 50, 75 y $100 \%$, respectivamente (Cuadro 1). Al respecto, el nitrógeno $(\mathrm{N})$ es el elemento más requerido por las plantas, ya que representa de 1 a $5 \%$ de la materia seca total, y es constituyente integral de proteínas, ácidos nucleicos, clorofila, coenzimas, fitohormonas y metabolitos secundarios (Hawkesford et al., 2012); asimismo, dosis óptimas de nitrógeno mejoran el proceso fotosintético, área foliar y materia seca total, factores que son determinantes para la producción de los cultivos (Leghari et al., 2016).

De acuerdo con los resultados encontrados, se sugiere emplear la solución nutritiva de Steiner a $100 \%$ en el cultivo de epazote, ya que presentó la mayor producción del dosel vegetal en comparación a 75 y 50 \% de concentración.

\section{Producción de epazote}

Hubo diferencias $(P \leq 0.05)$ en la producción de epazote fresco ( $\mathrm{g}$ por planta). Las plantas con la mayor producción se obtuvieron cuando se regaron con la solución Steiner a 100 $\%$ (Figura 9). Cabe señalar, que a medida que se incrementó la concentración de la solución nutritiva, también la producción por planta aumentó. Esta respuesta puede atribuirse a una mayor disponibilidad y aprovechamiento nutrimental, lo cual influyó directamente sobre la producción de la planta de epazote, como también se ha reportado en cilantro cultivado en tezontle y con solución nutritiva de Steiner (Cruz-Crespo et al., 2017). 


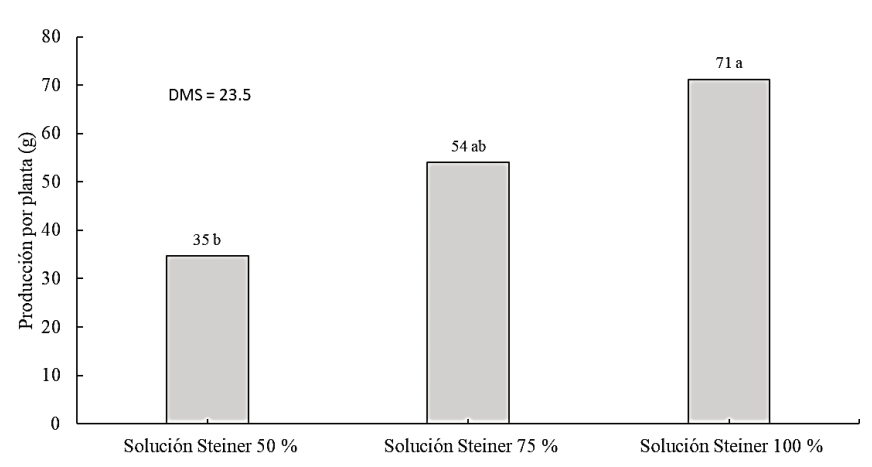

Figura 9. Producción por planta de epazote fresco en función de la concentración de la solución nutritiva de Steiner.

Figure 9. Plant production of fresh Mexican tea as a function of the Steiner nutrient solution concentration.

\section{CONCLUSIONES}

La mayor altura y producción en el cultivo de epazote cultivado en invernadero y con sustrato inorgánico se obtiene con la solución nutritiva de Steiner a $100 \%$, lo cual coincide con los valores más altos de la tasa de crecimiento del cultivo, la tasa absoluta de crecimiento y el índice de área foliar, y cuyas ecuaciones se ajustan a un modelo cuadrático. Con los resultados obtenidos, se sugiere emplear la solución nutritiva de Steiner a $100 \%$ para obtener la mejor productividad en esta planta aromática y medicinal.

\section{REFERENCIAS}

Aguilar Carpio, C., Juárez-López, P., Campos-Aguilar I.H., AliaTejacal, I., Sandoval-Villa, M. y López-Martínez, V. 2018. Análisis de crecimiento y rendimiento de uchuva (Physalis peruviana L.) cultivada en hidroponía e invernadero. Revista Chapingo Serie Horticultura. 24: 191-202.

Aguilar-Carpio, C., Escalante-Estrada, J.A.S. y Aguilar-Mariscal, I. 2015. Análisis de crecimiento y rendimiento de maíz en clima cálido en función del genotipo, biofertilizante y nitrógeno. Terra Latinoamericana 33: 51-62.

Azofeifa, Á., Moreira, M. 2004. Análisis de crecimiento del chile jalapeño (Capsicum annuum L. cv. Hot), en Alajuela, Costa Rica. Agronomía Costarricense. 28: 57-67.

Azcón-Bieto, J. y Talón, M. 2008. Fundamentos de Físiologia Vegetal (2a. ed.). McGraw-Hill. Madrid, España. 651 p.

Blanckaert, I., Paredes-Flores, M., Espinosa-García, F.J., Piñero, D. y Lira. R. 2012. Ethnobotanical, morphological, phytochemical and molecular evidence for the incipient domestication of Epazote (Chenopodium ambrosioides L.: Chenopodiaceae) in a semi-arid region of México. Genetic Resources and Crop Evolution. 59: 557-573.

Barraza, F.V., Fischer, G., Cardona, C.E. 2004. Estudio del proceso de crecimiento del cultivo del tomate (Lycopersicon esculentum Mill.) en el Valle del Sinú medio, Colombia Agronomía Colombiana. 22: 81-90.

Barrientos-Llanos, H., del-Castillo-Gutiérrez, C.R., GarcíaCárdenas, M. 2015. Análisis de crecimiento funcional, acumulación de biomasa y translocación de materia seca de ocho hortalizas cultivadas en Invernadero. Revista de Investigación e Innovación Agropecuaria y de Recursos Naturales. 2: 76-86.
Cruz-Crespo, E., Can-Chulim, A., Loera-Rosales, J., AguilarBenítez, G., Pineda-Pineda, J. y Bugarín-Montoya, R. 2017. Extracción de N-P-K en Coriandrum sativum "Pakistan" en hidroponía. Revista Mexicana de Ciencias Agrícolas. 8: 355367.

Del Pozo, P.P., Herrera, R.S., García, M., Cruz, A.M. y Romero, A. 2001. Análisis de crecimiento y desarrollo del pasto estrella con y sin adición de fertilizante nitrogenado. Revista Cubana de Ciencias Agrícolas. 35: 51-58.

Escalante-Estrada, A.S. y Kohashi-Shibata, J. 2015. El rendimiento y crecimiento del frijol. Manual para la toma de datos. Colegio de Postgraduados, Montecillo, México. 84 p.

Fghire, R., Anaya, F., Issa, O.A. y Wahbi, S. 2017. Physiological and growth response traits to water deficit as indicators of tolerance criteria between quinoa genotypes. Journal of Materials and Environmental Sciences. 8: 2084-2096.

Gómez-Castellanos, J.R. 2008. Epazote (Chenopodium ambrosioides L.). Revisión a sus características morfológicas, actividad farmacológica, y biogénesis de su principal principio activo, ascaridol. Boletín Latinoamericano y de Caribe de Plantas Medicinales y Aromáticas. 7: 3-9.

Graciano-Cristóbal, M.J., Sumaya-Martínez, M.T., RodríguezCarpena, J.G., Bautista-Rosales, P.U., Jiménez-Ruiz, E.I., Sánchez-Herrera, L.M., López-Nahuatt, G. 2020. Impacto en la formación de acrilamida a partir de aditivos de especias culinarias con actividad antioxidante. Biotecnia. 22: 128-135.

Hawkesford, M., Horst, W., Kichey, T., Lambers, H., Schjoerring, J., Moller, I.S. y White, P. 2012. Functions of macronutrients. In P. Marschner (Ed.), Marschners's Mineral Nutrition of higher plants (3rd ed., pp. 135-189). USA: Academic press.

Hernández-Córdova, N., Soto-Carreño, F., Plana-Llerena, R. 2015. Comportamiento del crecimiento y rendimiento del cultivo del trigo (Triticum aestivum L.) en tres fechas de siembra. Cultivos Tropicales. 36: 86-92.

Huidobro-Medina, E.S. 2013. Establecimiento del cultivo hidropónico y evaluación de actividad biológica de epazote (Teloxys ambrosioides Weber). Universidad Nacional Autónoma de México. Facultad de Estudios Superiores Iztacala. Los Reyes Iztacala, Estado de México. 76 p.

Méndez-Isidro, M. 2014. Distribución potencial del epazote (Chenopodium ambrosioides: Chenopodiaceae). Universidad Nacional Autónoma de México. Facultad de Estudios Superiores Iztacala. Los Reyes Iztacala, Estado de México. 33 p.

Jaramillo, B.E., Duarte, R.E. y Delgado, W. 2012. Bioactividad del aceite esencial de Chenopodium ambrosioides L. colombiano. Revista Cubana de Plantas Medicinales. 17: 54-64.

Juárez-Rosete, C.R., Aguilar-Castillo, J.A., Aburto-González, C.A. y Alejo-Santiago, G. 2019. Producción de biomasa, requerimiento nutrimental de nitrógeno, fosforo y potasio, y concentración de la solución nutritiva en orégano. Revista Chapingo Serie Horticultura. 25: 17-28.

Leghari, S.J., Wahocho, N.A., Laghari, G.M., Laghari, A.H., Bhabhan, G.M., Talpur, K.H., Bhutto, T.A., Wahocho, S.A. y Lashari, A.A. 2016. Role of nitrogen for plant growth and development: A review. Advances in Environmental Biology. 10: 209-218.

Nascimento, R.F.F., Cruz, G.V.B., Pereira, P.V.S., Maciel, M.C.G., Silva, L.A., Azevedo, A.P.S., Borroqueiro, E.S.B. y Guerra, R.N.M. 2006. Ascitic and solid Ehrlich tumor inhibition by Chenopodium ambrosioides L. treatment. Life Sciences. 78: 2650-2653. 
Rakesh, P., Vijay, P., Madurima, D., Mahesh, M. y Ramesh, C.M. 2017. Plant Growth Analysis. Manual of ICAR Sponsored Training Programme on "Physiological Techniques to Analyze the Impact of Climate Change on Crop Plant". 103$107 \mathrm{pp}$.

Sánchez-Torres, J. D., Ligarreto-Moreno, G.A. y Leiva-Barón, F.R. 2012. Variabilidad del crecimiento y rendimiento del cultivo de maíz para choclo (Zea mays L.) como respuesta a diferencias en las propiedades químicas del suelo en la sabana de Bogotá, Colombia. Revista Facultad Nacional de Agronomía Medellín. 65: 6579-6583.

Santos-Castellanos, M., Segura-Abril, M. y Ñústez-López, C.E. 2010. Análisis de crecimiento y relación fuente-demanda de cuatro variedades de papa (Solanum tuberosum L.) en el municipio de Zipaquirá (Cudinamarca, Colombia). Revista Facultad Nacional de Agronomía Medellín. 63: 5253-5266.
SAS Institute. 2004. SAS/STAT User's Guide. Release 9.1. SAS Institute. Cary, North Carolina, USA. 5121 p.

Steiner, A.A. 1984. The universal nutrient solution. In Proc. 6th International Congress on Soilless Culture (pp: 633-649). ISOSC. Wageningen, The Netherlands.

SIAP (Sistema de Información Agropecuaria). 2020. Sistema de Información Agropecuaria de Consulta. SAGARPA. México. https://nube.siap.gob.mx/cierreagricola/.

Taiz, L., E. Zeiger, I. M. Moller and A. Murphy. 2014. Plant Physiology \& Development. Sixth Edition. Sinauer Associates, Sunderland, MA, USA. $756 \mathrm{p}$.

Vidal-Gutiérrez, M., Torres-Moreno, H., Velázquez-Contreras, C.A., Rascón-Valenzuela, L.A., Robles-Zepeda, E. 2020. Actividad antioxidante y antiproliferativa de seis plantas medicinales del noroeste de México. Biotencia. 22: 40-45. 